\title{
Is the nasal optic disc sector important for morphometric glaucoma diagnosis?
}

\author{
J B Jonas, W M Budde
}

Br J Ophthalmol 2002;86:1232-1235

Background/aim: Since the central retinal vessel trunk usually located in the nasal optic disc sector can render difficult the delineation of the neuroretinal rim and optic disc, the aim of this study was to evaluate whether the nasal region of the optic nerve head is important, or can be left out, for the morphometric glaucoma diagnosis.

Methods: The clinical observational study included 1337 patients with primary or secondary open angle glaucoma and 649 normal subjects. The glaucoma group was divided into 1187 patients with glaucomatous visual field defects ("perimetric glaucoma"), and into 150 patients with optic nerve head changes and normal visual fields ("preperimetric glaucoma"). Colour stereo optic disc photographs were morphometrically evaluated.

Results: Highest diagnostic power for the separation between the normal group and the perimetric glaucoma group, and for the differentiation between the normal group and the preperimetric glaucoma group, had the sum of inferotemporal rim area plus superotemporal rim area, the sum of inferotemporal rim area plus superotemporal rim area plus temporal rim area, and the inferotemporal rim area as single parameter. The lowest diagnostic precision had the nasal rim area as single parameter or in combination with rim measurements in other disc sectors.

Conclusion: Excluding the nasal optic disc sector does not markedly decrease the diagnostic power of morphometric optic disc analysis in glaucoma diagnosis. It may have importance for an automated computerised morphometric detection of glaucomatous optic nerve damage.

n the morphometric analysis of the optic nerve head, outlining of the optic disc and optic cup is of utmost importance to determine the size and shape of the neuroretinal rim which is one of the main targets in the quantitative and qualitative assessment of the optic nerve head. ${ }^{1}$ Because of the anatomy of the optic nerve head, the border of the optic disc can more easily be detected in the temporal, inferior, and superior regions than in the nasal region. In a parallel manner, it is easier to demarcate the inner margin of the neuroretinal rim in the inferior and superior disc regions than in the nasal disc sector, where the central retinal vessel

Table 1 Composition of the study groups (mean (SD))

\begin{tabular}{lll}
\hline & Glaucoma group & Normal group \\
\hline Number & 1337 & 649 \\
Women/men & $668 / 669$ & $328 / 321$ \\
Age (years) & $59.67(15.47)$ & $45.40(15.05)$ \\
Refractive error (D) & $-0.28(1.31)$ & $-0.43(1.88)$ \\
Range (D) & -7.88 to +3.63 & -7.50 to +7.00 \\
\hline
\end{tabular}

trunk and the major retinal vessels are attached to, or lie upon, the neuroretinal rim and its border with the optic cup. Since difficulties in outlining the margins of the optic disc and optic cup will lead to an inaccuracy in measuring the neuroretinal rim, it was the aim of this study to determine how important the assessment of the nasal sector of the optic disc is for the detection of glaucomatous optic nerve damage, and whether the nasal disc region can be left out in the quantitative analysis of the optic nerve head in the diagnosis of glaucoma.

\section{PATIENTS AND METHODS}

The clinical observational study included 1337 patients with primary or secondary open angle glaucoma, and 649 normal subjects (Table 1). Only one randomly selected eye per subject and patient was taken for the statistical analysis. Patients with a highly myopic refractive error equal to or higher than -8 dioptres were excluded because of differences in the anatomy of the optic nerve head. ${ }^{2}$ The methods applied in the study adhered to the tenets of the declaration of Helsinki for the use of human subjects in biomedical research. Institutional review board/ethics committee approval was not required for this study.

All glaucoma patients had an open anterior chamber angle and were consecutively evaluated. The total glaucoma group was differentiated into a "perimetric" group and a "preperimetric" group. In the perimetric group, glaucomatous visual field defects were present. A glaucomatous visual field defect was defined as an Octopus Gl field with one of the following (a) at least three adjacent test points having a deviation of equal to or greater than $5 \mathrm{~dB}$ and with one test point with a deviation greater than $10 \mathrm{~dB}$ lower, (b) at least two adjacent test points with a deviation equal to or greater than $10 \mathrm{~dB},(\mathrm{c})$ at least three adjacent test points with a deviation equal to or greater than $5 \mathrm{~dB}$ abutting the nasal horizontal meridian, or (d) a mean visual field defect of more than $2 \mathrm{~dB}$. Rate of false positive answers and rate of false negative answers had to be equal to or less than $15 \%$. In the preperimetric group, visual field was normal, and glaucomatous changes of the optic nerve head were present, such as an unusually small neuroretinal rim area in relation to the optic disc size, cup:disc diameter ratios being vertically higher than horizontally, and localised or diffuse retinal nerve fibre layer defects.

The perimetric group consisted of 1187 patients with a mean age of 57.7 (SD 15.7) years and a mean refractive error of -0.30 ( 1.58 ) dioptres. The preperimetric group included 150 patients with a mean age of 46.8 (13.8) years and a mean refractive error of -0.68 (2.02) dioptres. The total glaucoma group $(n=1337)$ included eyes with primary open angle glaucoma, pseudoexfoliation glaucoma, pigmentary glaucoma, and eyes with normal pressure glaucoma $(n=118)$. Criteria for the diagnosis of normal pressure glaucoma were maximal intraocular pressure readings equal to or less than $21 \mathrm{~mm} \mathrm{Hg}$ in at least two 24 hour pressure profiles obtained by slit lamp applanation tonometry and containing measurements at 5 pm, 9 pm, midnight, 7 am, and noon. Ophthalmoscopy, medical history, and neuroradiological, neurological, and medical 
Table 2 Sensitivity at a prefixed specificity of $80 \%$ and at a prefixed specificity of $95 \%$ to separate normal subjects from preperimetric glaucoma patients, and to separate normal subjects from perimetric glaucoma patients

\begin{tabular}{|c|c|c|c|c|c|c|}
\hline & \multirow[b]{2}{*}{$\begin{array}{l}\text { Sensitivity at } \\
\text { specificity } 80 \%\end{array}$} & \multicolumn{2}{|c|}{$\begin{array}{l}\text { Normal group/preperimetric } \\
\text { glaucoma }\end{array}$} & \multirow[b]{2}{*}{$\begin{array}{l}\text { Sensitivity at } \\
\text { specificity } 80 \%\end{array}$} & \multicolumn{2}{|c|}{$\begin{array}{l}\text { Normal group/perimetric } \\
\text { glaucoma }\end{array}$} \\
\hline & & $\begin{array}{l}\text { Sensitivity at } \\
\text { specificity } 95 \%\end{array}$ & ROC area & & $\begin{array}{l}\text { Sensitivity at } \\
\text { specificity } 95 \%\end{array}$ & ROC area \\
\hline \multicolumn{7}{|l|}{ Neuroretinal rim area in the } \\
\hline Temporal inferior sector & $54 \%$ & $30.7 \%$ & $74.5 \%$ & $76.8 \%$ & $56.3 \%$ & $84.8 \%$ \\
\hline Temporal superior sector & $46.0 \%$ & $20.0 \%$ & $70.5 \%$ & $67.6 \%$ & $47.3 \%$ & $80.4 \%$ \\
\hline Temporal horizontal sector & $39.3 \%$ & $20.7 \%$ & $59.5 \%$ & $64.4 \%$ & $45.3 \%$ & $81.2 \%$ \\
\hline Nasal sector & $27.3 \%$ & $12.7 \%$ & $62.5 \%$ & $21.7 \%$ & $10.4 \%$ & $65.7 \%$ \\
\hline \multicolumn{7}{|l|}{ Sum of sector: } \\
\hline Temporal inferior + temporal superior & $57.3 \%$ & $27.3 \%$ & $74.5 \%$ & $75.4 \%$ & $52.5 \%$ & $84.3 \%$ \\
\hline $\begin{array}{l}\text { Temporal inferior +temporal superior + } \\
\text { temporal }\end{array}$ & $55.3 \%$ & $22.0 \%$ & $72.9 \%$ & $78.3 \%$ & $57.8 \%$ & $86.9 \%$ \\
\hline $\begin{array}{l}\text { Temporal inferior + temporal superior + } \\
\text { nasal }\end{array}$ & $39.3 \%$ & $22.0 \%$ & $69.4 \%$ & $60.9 \%$ & $39.8 \%$ & $78.4 \%$ \\
\hline Total rim area & $56.0 \%$ & $21.3 \%$ & $74.1 \%$ & $72.1 \%$ & $52.5 \%$ & $83.5 \%$ \\
\hline
\end{tabular}

examinations did not reveal any other reason, such as intrasellar or suprasellar tumours, retinal vessel occlusions, optic disc drusen, or non-arteritic anterior ischaemic optic neuropathy for optic nerve damage than glaucoma.

Colour stereo optic disc transparencies (15 degrees) had been taken for all eyes, using a Zeiss telecentric fundus camera $\left(30^{\circ}\right.$ fundus camera, equipped with a $15^{\circ}$ converter; Zeiss, Oberkochem, Germany). The optic disc slides were projected in a scale of 1 to 15 . The outlines of the optic cup, optic disc, and peripapillary scleral ring were plotted on paper and morphometrically analysed. To obtain values in absolute size units - that is, $\mathrm{mm}$ or $\mathrm{mm}^{2}$, the ocular and photographic magnification was corrected using the Littmann method. ${ }^{3}$ The optic cup was defined on the basis of contour and not of pallor. The border of the optic disc was identical with the inner side of the peripapillary scleral ring. To compare various optic disc regions with each other, the intrapapillary region was divided into four sectors. The temporal superior sector and the temporal inferior disc sector were right angled and were tilted $15^{\circ}$ temporal to the vertical optic disc axis. The two other sectors covered the remaining area: the temporal horizontal sector was 60 degrees wide, and the nasal disc sector covered $120^{\circ}$. In each of the four sectors, size of the neuroretinal rim and optic cup were measured separately. The method has already been described in detail. ${ }^{4}$

Descriptive statistical analysis included means and standard deviations. Confirmative analyses used a significance level of 0.05 (two sided). Correlation analyses used the Pearson's correlation coefficient. All statistical analyses were performed using spsswin (release 9.0).

\section{RESULTS}

\section{Diagnostic power}

Highest diagnostic power, expressed as sensitivity and specificity, for the separation between the normal subjects and the glaucoma patients with visual field defects had the sum of the inferotemporal rim area plus the superotemporal rim area plus the temporal rim area, the inferotemporal rim area as single parameter, and the sum of the inferotemporal rim area plus the superotemporal rim area, followed by the total rim area and the superotemporal rim area as single parameter. The diagnostic precision was lowest for the nasal rim area, and the sum of the inferotemporal rim area plus the superotemporal rim area plus the nasal rim area (Table 2 ).

Correspondingly, the area under the receiver operator curve (ROC) was largest for the sum of the inferotemporal rim area plus the superotemporal rim area plus the temporal rim area, the inferotemporal rim area as single parameter, and the sum of the inferotemporal rim area plus the superotemporal rim area (Table 2). The area under the ROC is a measure for the diagnostic precision of a parameter in separating two study groups from each other. It combines the figures of specificity and sensitivity. A value of $100 \%$ is the best possible diagnostic precision associated with a $100 \%$ separation of the study groups. A value of $50 \%$ means a random distribution of the subjects included in the study. The area under the ROC was smallest for the nasal rim area as single parameter and for the sum of the inferotemporal rim area plus the superotemporal rim area plus the nasal rim area (Table 2).

Similar data were found for the separation between the normal group and the preperimetric group. Highest diagnostic

Table 3 Coefficients of the correlations between neuroretinal rim area measurements and the mean visual field defect in the whole study population

\begin{tabular}{lll}
\hline & $\begin{array}{l}\text { Correlation } \\
\text { coefficient }\end{array}$ & $p$ Value \\
\hline Neuroretinal rim area & -0.58 & $<0.001$ \\
$\quad$ Temporal inferior sector & -0.55 & $<0.001$ \\
$\quad$ Temporal superior sector & -0.43 & $<0.001$ \\
Temporal sector & -0.34 & $<0.001$ \\
Nasal sector & & \\
& -0.59 & $<0.001$ \\
Temporal inferior + temporal superior sector & -0.60 & $<0.001$ \\
Temporal inferior +temporal superior + temporal sector & -0.53 & $<0.001$ \\
$\quad$ Temporal inferior + temporal superior + nasal sector & -0.59 & $<0.001$ \\
Total rim area & & \\
\hline
\end{tabular}


precision had the sum of the inferotemporal rim area plus the superotemporal rim area, followed by the sum of the inferotemporal rim area plus the superotemporal rim area plus the temporal rim area, followed the inferotemporal rim area as single parameter (Table 2). The lowest diagnostic precision had the nasal rim area as single parameter, the temporal horizontal rim area, and the sum of the inferotemporal rim area plus the superotemporal rim area plus the nasal rim area.

The area under the ROC was largest for the sum of the inferotemporal rim area plus the superotemporal rim area plus the temporal rim area, the inferotemporal rim area as single parameter, and the sum of the inferotemporal rim area plus the superotemporal rim area (Table 2). It was smallest for the nasal rim area as single parameter, and for the sum of the inferotemporal rim area plus the superotemporal rim area plus the nasal rim area (Table 2).

\section{Correlation coefficients}

The coefficients of the correlations between neuroretinal rim area measurements in the various optic disc sectors and the mean visual field defect were highest for the sum of the inferotemporal rim area plus the superotemporal rim area plus the temporal rim area, the sum of the inferotemporal rim area plus the superotemporal rim area, the total rim area, and the inferotemporal rim area as single parameter (Table 3). The correlation coefficients were lowest for the relation between the mean visual field defects and the nasal rim area (Table 3 ).

\section{DISCUSSION}

The results suggest that examination of the nasal region of the optic nerve head compared with the assessment of other regions of the optic disc has less importance in detecting glaucomatous optic nerve damage. In the present study, diagnostic precision in the separation of glaucoma patients and normal subjects was highest for the inferotemporal neuroretinal rim area, the sum of the inferotemporal rim area plus the superotemporal rim area, the sum of the inferotemporal rim area plus the superotemporal rim area plus the temporal rim area, and the superotemporal rim area (Table 2). The diagnostic precision was lowest for the nasal rim area taken as single parameter, followed by sums of neuroretinal rim areas including the nasal disc sector. The data suggest that exclusion of the nasal optic disc sector increased sensitivity and specificity of the neuroretinal rim area measurements to detect glaucomatous optic nerve damage.

This finding may have clinical importance. Firstly, for the diagnosis of glaucoma, it may be better to concentrate on the inferotemporal, superotemporal, and temporal sectors and, in case of doubt, to neglect the nasal optic disc sector. The importance of the inferotemporal disc sector and superotemporal disc sector for the diagnosis of glaucoma has already been shown in numerous studies, ${ }^{5-7}$ while the lesser importance of the nasal optic disc sector for the detection of glaucoma has not been clearly demonstrated yet. Secondly, exclusion of the nasal optic disc sector will not decrease the diagnostic precision of the optic disc evaluation in the diagnosis of glaucoma. It may be important for the fully automatic assessment of the appearance of the optic nerve head if future studies, using the confocal laser scanning tomographic technique for assessment of the optic nerve head, confirm the results of the present study. For confocal laser scanning tomography of the optic nerve head, the border of the optic disc has to be interactively outlined by an examiner. Because of the location of the central retinal vessel trunk in the nasal part of the optic nerve head, and the often partially oblique insertion of the optic nerve into the sclera, outlining of the optic disc border is easier in the temporal, inferotemporal, and superotemporal disc sectors than in the nasal disc sector. If the nasal optic disc sector can be left out of the morphometric optic nerve head assessment, outlining of the disc border on the confocal laser scanning tomographic images will become easier, and the examination dependent variation of the optic disc outlining will have less impact on the optic nerve head measurements. Assuming that the optic disc border in the disc regions outside of the nasal disc sector will be determined automatically by using a short wavelength laser such as the laser used for confocal laser scanning fluorescein angiography, and excluding the nasal optic disc region out of the optic nerve head analysis, may perhaps result in a fully automatic morphometric optic nerve head examination in which it will no longer be necessary to manually outline the border of the optic nerve head.

There are limitations to the present study. For some patients, the diagnosis of glaucoma was partially based on the appearance of the optic nerve head. Since nasal cupping of the optic disc has not traditionally been considered to be a classic aspect of glaucomatous optic nerve damage, one cannot exclude the possibility that if there were patients with glaucoma in whom nasal cupping was the predominant part of their optic nerve damage, and if there were no glaucomatous visual field defects, the patients would not have been selected for the study, simply because they probably would not have been considered glaucomatous to start with. Therefore, the fact that nasal cupping was not found to be an important characteristic of patients with glaucoma, may partially reflect a flaw in the method of patient selection. For the definition of glaucomatous optic nerve damage, however, the main parameters were an unusual shape of the neuroretinal rim and defects in the retinal nerve fibre layer. The latter is independent of whether or not the nasal sector of the optic disc was the one where the damage was located. For the configuration of the neuroretinal rim, any deviation of the so called ISN'T rule, in which the smallest part of the neuroretinal rim should be located in the temporal disc sector was considered to be suggestive of glaucoma. One may infer that a loss of rim in the nasal disc sector may also have been considered to be abnormal and taken as a sign for glaucomatous optic nerve damage. Additionally, the majority (1187 of 1337 patients) of the patients included in the study showed glaucomatous visual field defects so that they would have been included into the study already solely based on the result of their perimetric examination.

The results of the present study may not serve to generally exclude the nasal optic disc region from the assessment of the optic nerve head. Certainly in advanced glaucoma, the nasal sector is often all that is left to evaluate. Additionally, qualitative parameters such as the presence of optic disc haemorrhages have to be examined in all regions of the optic disc. Furthermore, the present study has not evaluated the importance of the nasal optic disc sector for the follow up examination of eyes with glaucoma, ${ }^{8}$ so that the results of the present study are valid only for the detection of glaucomatous optic nerve damage.

In summary, the nasal optic disc sector may be left out for the morphometric optic nerve head analysis in the detection of glaucoma, what may have importance for the clinical ophthalmoscopy as well as for the development of a fully automated quantitative analysis of the optic disc.

\section{ACKNOWLEDGEMENT}

Supported by Deutsche Forschungsgemeinschaft DFG (SFB 539) (Bonn; Germany)

Proprietary interest: none

\section{Authors' affiliations}

J B Jonas, W M Budde, Department of Ophthalmology and Eye Hospital, University Erlangen-Nürnberg, and Faculty of Clinical Medicine Mannheim, University of Heidelberg, Mannheim, Germany 
Correspondence to: $\mathrm{Dr} J$ Jonas, Universitäts-Augenklinik, Theodor-Kutzer-Ufer 1-3, 68167 Mannheim, Germany; Jost.Jonas@augen.ma.uni-heidelberg.de

Accepted for publication 7 June 2002

\section{REFERENCES}

1 Jonas JB, Budde WM, Panda-Jonas S. Ophthalmoscopic evaluation of the optic nerve head. Surv Ophthalmol 1999;43:293-320.

2 Jonas JB, Gusek GC, Naumann GOH. Optic disk morphometry in high myopia. Graefes Arch Clin Exp Ophthalmol 1988;226:587-90.

3 Littmann H. Zur Bestimmung der wahren Größe eines Objektes auf dem Hintergrund des lebenden Auges. Klin Monatsbl Augenheilkd 1982;180:286-9.
4 Jonas JB, Gusek GC, Naumann GOH. Optic disc, cup and neuroretinal rim size, configuration, and correlations in normal eyes. Invest Ophthalmol Vis Sci 1988;29:1 15-8; correction: Invest Ophthalmol Vis Sci 1991;32:1893.

5 Pederson JE, Anderson DR. The mode of progressive disc cupping in ocular hypertension and glaucoma. Arch Ophthalmol 1980;98:490-5. 6 Caprioli J, Miller JM, Sears M. Quantitative evaluation of the optic nerve head in patients with unilateral visual field loss from primary open-angle glaucoma. Ophthalmology 1987;94:1484-7.

7 Garway-Heath DF, Hitchings RA. Quantitative evaluation of the optic nerve head in early glaucoma. BrJ Ophthalmol 1998;82:352-61.

8 Chauhan BC, McCormick TA, Nicolela MT, et al. Optic disc and visual field changes in a prospective longitudinal study of patients with glaucoma: comparison of scanning laser tomography with conventional perimetry and optic disc photography. Arch Ophthalmol 2001;119:1492-9

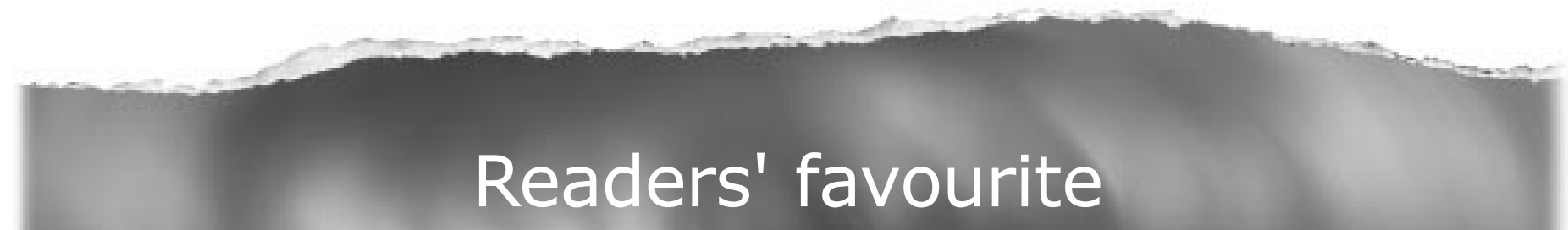

\section{Top 10}

Click on the "Top 10" button on the homepage to see which are the best read articles each month

www.bjophthalmol.com 